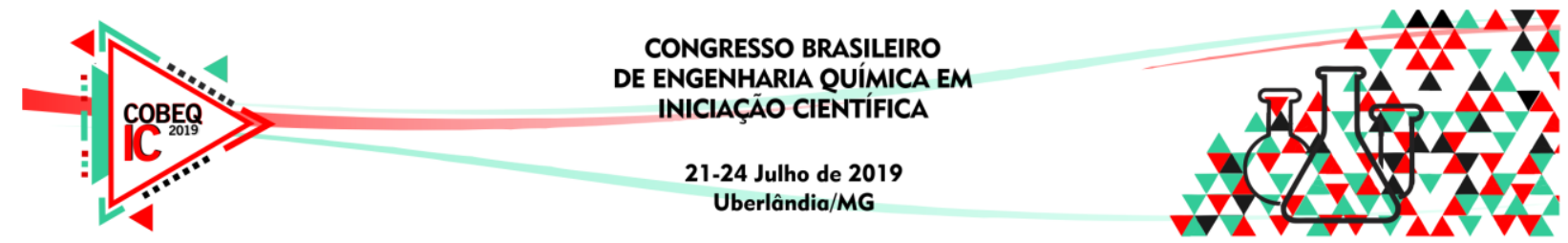

\title{
ESTUDO NUMÉRICO E TEÓRICO DA PERDA DE CARGA EM UM TROCADOR DE CALOR DE DUPLO TUBO PARA CONDIÇÕES ISOTÉRMICAS E DE REGIME PERMANENTE
}

\author{
M. Y. CARDOSO ${ }^{1}$, G. C. LOPES ${ }^{2}$, G. H. JUSTI ${ }^{1}$ \\ ${ }^{1}$ Universidade Federal de Mato Grosso, Campus Universitário de Várzea Grande, Faculdade \\ de Engenharia \\ ${ }^{2}$ Universidade Federal de São Carlos, Departamento de Engenharia Química \\ E-mail para contato: mthsyr@outlook.com
}

\begin{abstract}
RESUMO - Os trocadores de calor de tubo duplo (TCDT) são equipamentos de extrema importância e amplamente utilizados em diversos setores industriais. O equipamento está presente em diversas etapas dos processos industriais e sua eficiência baseiam-se no aumento de troca térmica e uma baixa perda de carga. $\mathrm{O}$ presente trabalho teve como abordagem uma análise do comportamento computacional e teórico referente a perda de carga em um TCDT isotérmico, analisando também diferentes regimes de escoamento com propriedade constante dos fluidos. As simulações realizadas se demonstraram eficazes nos resultados obtidos das perdas de carga, apresentando desvio padrão satisfatórios, principalmente para o tubo. Assim, a CFD apresentou ser uma ferramenta viável para a obtenção dos resultados e também para futuras modificações geométricas no equipamento.
\end{abstract}

\section{INTRODUÇÃO}

Os trocadores de calor são equipamentos indispensáveis na engenharia e indústrias químicas, uma vez que estes são responsáveis pelas transferências de calor entre dois ou mais fluidos sem que os mesmos entrem em contato. Em razão da diversidade, esses equipamentos são normalmente classificados de acordo com seu formato de construção, que são desenvolvidos visando uma alta eficiência de troca térmica.

Um exemplo de trocador de calor com esses requisitos, é o trocador de calor de duplo tubo (TCDT), que é constituído dois cilindros concêntricos cujos sentidos de vazão podem ser em contracorrente ou em paralelo. Para este equipamento o tubo anular (externo) é, usualmente, destinado um fluido de alta capacidade calorifica, enquanto que o tubo de produto (interno) é destinado ao fluido de interesse. Este equipamento é considerado um dos mais utilizados por diversos setores industriais, em razão de sua fácil construção e manutenção, além de ser amplamente utilizado na reutilização de energia térmica, na pasteurização de alimentos com alta ou média viscosidade e, em recentes estudos, no escoamento de nanopartículas sólidas em suspensão, ou nanofluídos, uma vez que estas aumentam significativamente a eficiência de troca térmica entre os fluidos (GOODARZI et al., 2016; OMIDI et al., 2017). 


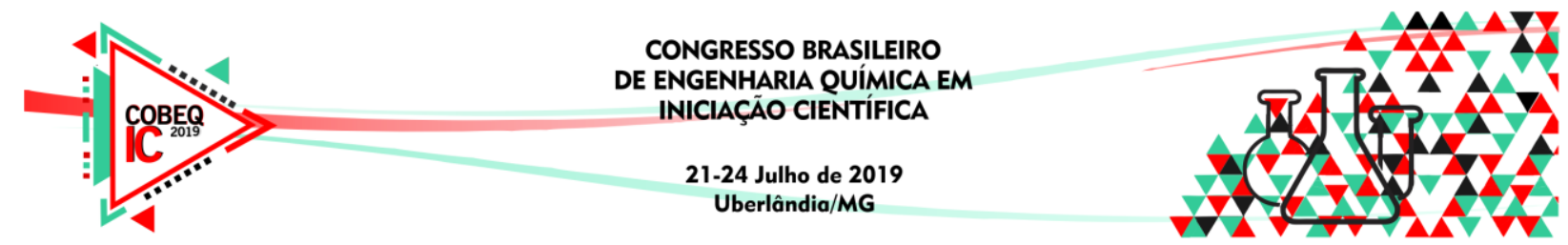

Na literatura é possível encontrar estudos sobre os trocadores de calor de duplo tubo com diferentes configurações, como o trabalho relatado por Wang et al. (2019). Aonde os mesmos analisaram, numericamente, trocadores de calor de tubo duplo com congruentes helicoidais, e baseando-se no coeficiente de convecção, queda de pressão e na eficiência energética. Os autores determinaram uma condição de dimensões que maximiza a troca térmica sem resultar em uma queda de pressão elevada. Estudos como este são comuns na literatura, uma vez que ao dimensionar parâmetros que aumentam de transferência de calor, esses acarretam indiretamente em uma elevada perda de carga, o que gera altos custos em escalas industriais com o bombeamento dos fluidos (YADAV \& SAHU, 2019). Um dos principais fatores que aumentam significativamente os custos de operação em uma indústria é a perda de carga, ou em casos que não há compressão ou expansão, a queda de pressão. Esta refere-se à pressão de um fluido que será perdida por atrito com as paredes durante sua condução ao longo de uma tubulação. E uma vez que é de interesse evitar diminuição das vazões ao longo da produção, o ideal é que a pressão fornecida para a manter o fluxo do fluido deve compensar a perda pela queda de pressão, ou seja, deve ser maior (SWAMEE et al., 2008).

Atualmente, essas perdas de cargas podem ser determinadas numericamente por softwares capazes de descrever resultados experimentais com alta eficiência e diminuindo o tempo e custos com análises experimentais, para muitos casos de engenharia. Entre os softwares destinados para análise de fluidodinâmica computacional (CFD), o software CFX apresenta várias funções inclusive a capacidade de avaliação de perfis de velocidade e pressão ao longo de uma determinada geometria, além da determinação de troca térmica entre fluidos utilizando equações diferenciais capazes de descrever o comportamento real com alta precisão.

Com a importância da análise de perda de carga e da sua dependência direta com o número de Reynolds em diferentes regimes de escoamento, o presente trabalho teve por objetivo a determinação da queda de pressão e do perfil de velocidade no TCDT, utilizando o software CFX, e comparar os resultados numéricos obtidos com equações matemáticas dispostas na literatura.

\section{METODOLOGIA NUMÉRICA}

\subsection{Considerações do modelo}

O desenvolvimento do presente trabalho teve como considerações:

- As propriedades dos fluidos são constantes;

- Os fluidos são isotérmicos, isto é, não há troca térmica entre o tubo do produto e o tubo anular;

- A água foi utilizada tanto no tubo quanto na parte anular como fluido de escoamento em contracorrente, e suas propriedades estão descritas na Tabela 1.

A perda de carga foi analisada comparando três vazões diferentes cujos valores de Reynolds se encontram na região laminar com valor inferior à 2300, na região turbulenta com valor superior à 3000 , na região intermediária com valor entre as situações anteriores. 


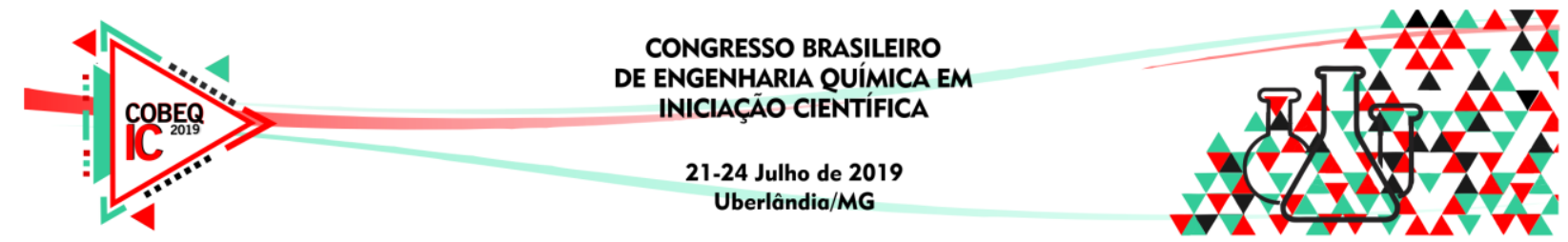

Tabela 1 - Propriedades dos fluidos de trabalho.

\begin{tabular}{|c|c|c|c|c|c|c|}
\hline Tubos & $\begin{array}{c}\text { Temperatura } \\
\left({ }^{\circ} \mathrm{C}\right)\end{array}$ & $\begin{array}{c}\text { Viscosidade } \\
\text { (cP) }\end{array}$ & $\begin{array}{l}\text { Densidade } \\
\left(\mathrm{kg} / \mathrm{m}^{3}\right)\end{array}$ & $\begin{array}{l}\text { Diâmetro } \\
\text { (m) }\end{array}$ & $\begin{array}{c}\text { Rugosidade } \\
\text { absoluta (m) } \\
10^{-5} \\
\end{array}$ & $\begin{array}{l}\text { Comprimento do } \\
\text { módulo }(\mathrm{m})\end{array}$ \\
\hline Produto & 30 & 0,8149 & 992,81 & 0,009 & \multirow{2}{*}{4,5} & \multirow{2}{*}{1,68} \\
\hline Anular & 65 & 0,4426 & 977,14 & $0,009 *$ & & \\
\hline
\end{tabular}

${ }^{*}\left(\mathrm{De}=0,021 ; \mathrm{Di}=0,012 ; \mathrm{D}_{\mathrm{h}}=\mathrm{D}_{\mathrm{e}}-\mathrm{D}_{\mathrm{i}}\right)$.

A perda de carga teórica foi determinada considerando o fator de atrito $\left(f_{f}\right)$, que é dependente da rugosidade absoluta do material $(e)$, do diâmetro da tubulação $(D)$ e do número de Reynolds (Re). Este fator é amplamente discutido na literatura, e pode ser determinado através da análise do gráfico de Moody, que correlaciona o fator de atrito experimental para diferentes números de Reynolds, no entanto quando deseja-se determinar analiticamente o fator de atrito utiliza-se a equação de Colebrook apresentada nas Equações 1 a 4 a seguir (WELTY et al., 2017):

- Escoamento laminar (Caso 1, $\mathrm{Re}=1450)$ :

$$
f_{f}=\frac{16}{\operatorname{Re}}
$$

- Escoamento de transição (Caso 2, $\operatorname{Re}=2500)$ :

$$
\frac{1}{f_{f}}=4 \cdot \log _{10}\left(\frac{D}{e}\right)+2,28-4 \cdot \log _{10}\left(4,67 \cdot \frac{D / e}{\operatorname{Re} \sqrt{f_{f}}}+1\right)
$$

- Escoamento turbulento com rugosidade (Caso 3, $\mathrm{Re}=4400)$ :

$$
\frac{1}{f_{f}}=4 \cdot \log _{10}\left(\frac{D}{e}\right)+2,28
$$

- Escoamento turbulento sem rugosidade $(\mathrm{Caso} 4, \mathrm{Re}=4400)$ :

$$
\frac{1}{f_{f}}=4 \cdot \log _{10}\left(\operatorname{Re} \cdot \sqrt{f_{f}}\right)-0,40
$$

\subsection{Geometria e malha}

A criação da geometria e da malha, e as simulações foram realizadas no software da CFX 14.5, licenciado pelo Departamento de Engenharia Química da Universidade Federal de São Carlos. A geometria (Figura 1) foi desenvolvida baseando-se em um trocador de calor real dividido em quatro módulos de troca térmica, que industrialmente visam uma diminuição do espaço exigido para sua construção e sendo que os resultados obtidos são proporcionais para uma quantidade maior de módulos. 


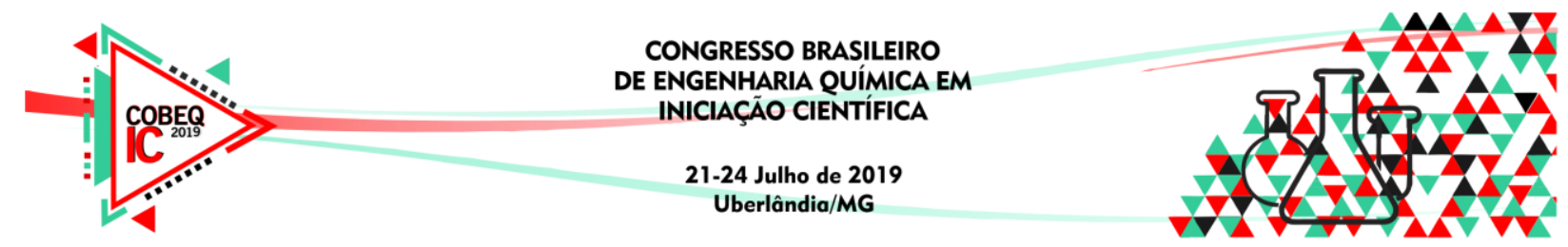

Figura 1 - Geometria e malha do TCDT para domínios anular e de produto.

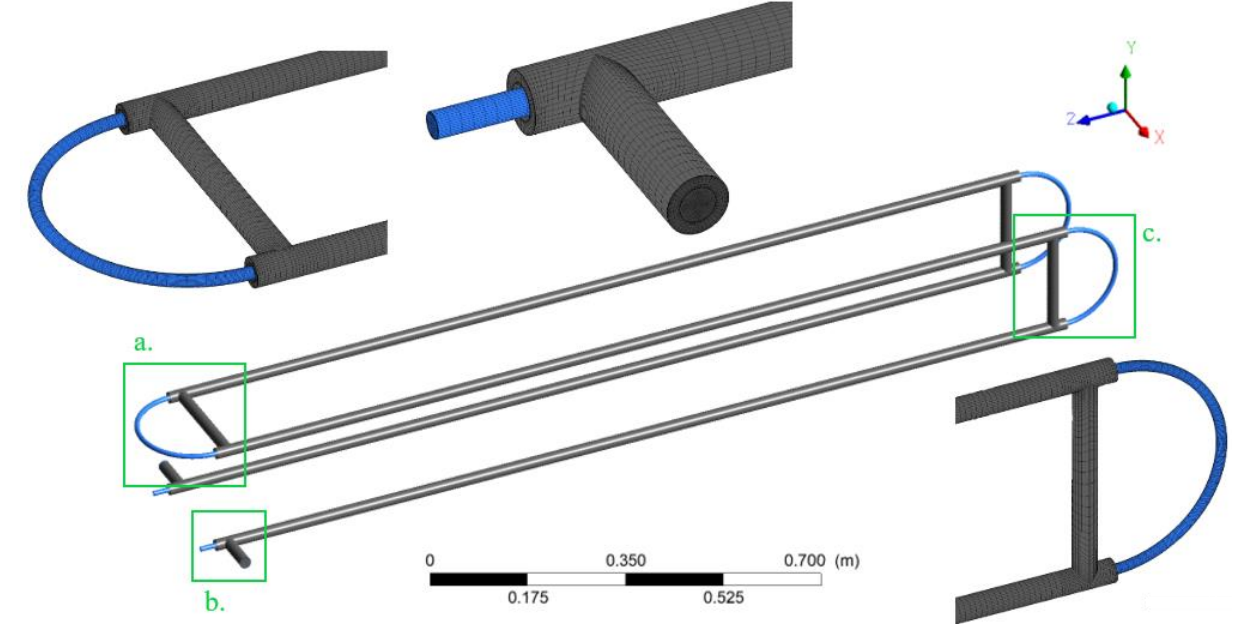

Enquanto que a malha desenvolvida é constituída completamente por elementos hexaédricos com refinos nas ligações e nas paredes, Figura 1, com número total de elementos de 1.468 .400 .

\section{RESULTADOS E DISCUSSÕES}

Os perfis de velocidade para o tubo anular nos três casos, Figura 2, possuem um comportamento quase que constante ao longo do TCDT, havendo pequenas variações principalmente nas regiões de localização dos acessórios em razão da variação do diâmetro seção transversal. Sendo, também, possível observar as regiões de fluido estagnado (nas extremidades), que em situações reais onde há troca térmica entre os fluidos, essa estagnação pode acarretar em incrustação nas paredes, dependendo do fluido de utilidade.

Figura 2 - Perfis de velocidade para os casos a) escoamento laminar, b) escoamento transiente e c) escoamento turbulento.

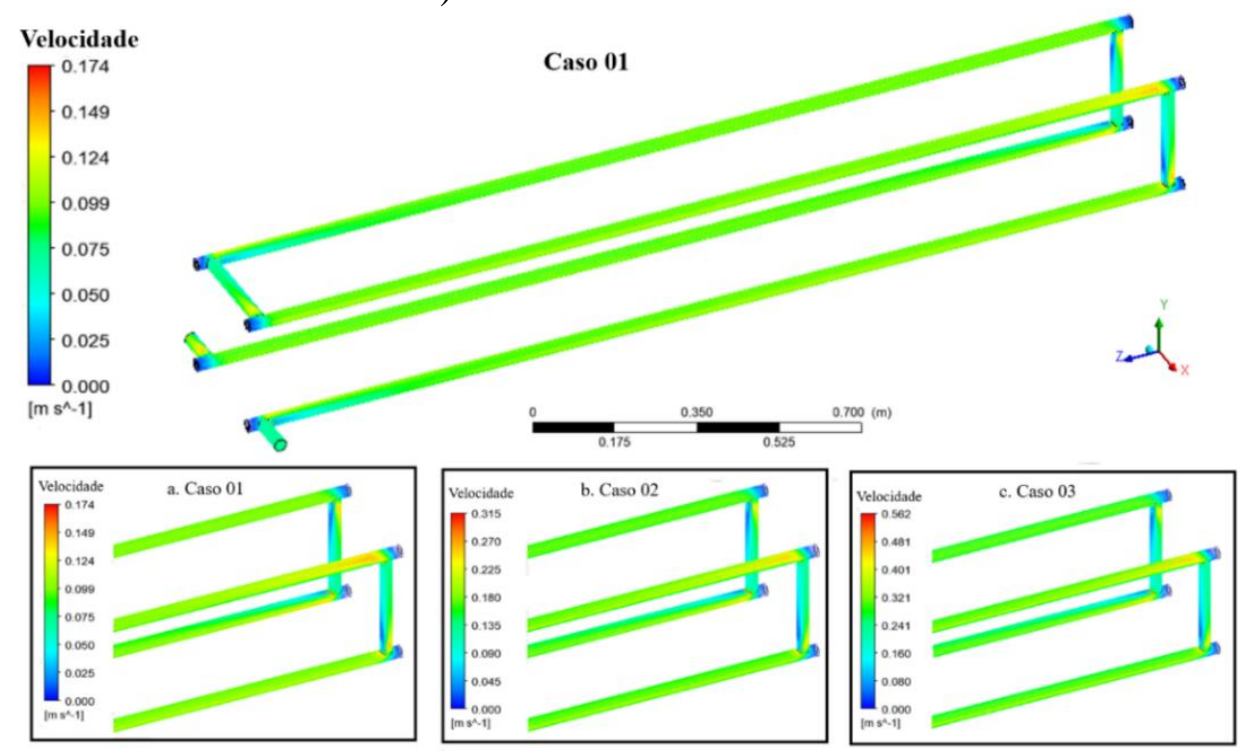



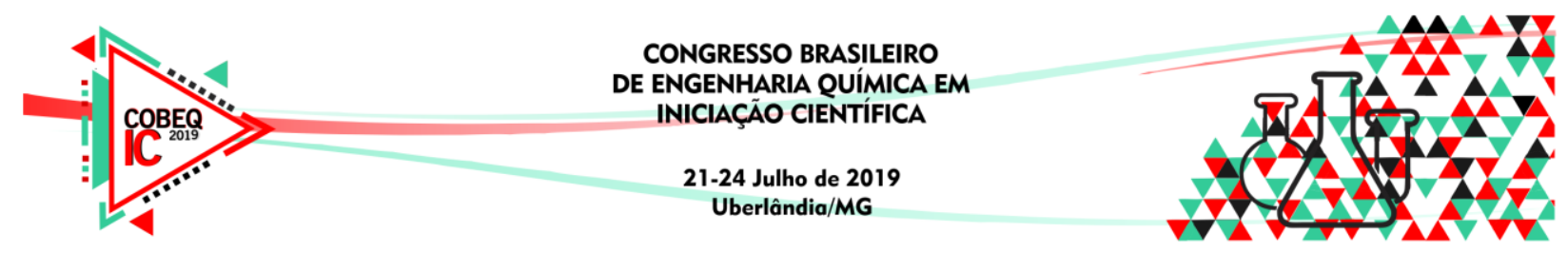

Os perfis de pressão, representados pela Figura 3, como esperado não são constantes ao longo do TCDT, havendo perdas de energia principalmente por atrito do fluido com as paredes dos tubos, além de perdas localizadas referente à diferença de cotas e de mudanças repentinas da direção do fluido. É possível observar nos três casos uma maior queda de pressão para o tubo de produto quando comparado ao tubo anular, sendo associada principalmente por uma vazão superior, uma vez que este possui uma temperatura muito menor e consequentemente uma maior viscosidade.

Figura 3 - Perfis de pressão para os casos a) escoamento laminar, b) escoamento transiente e c) escoamento turbulento.

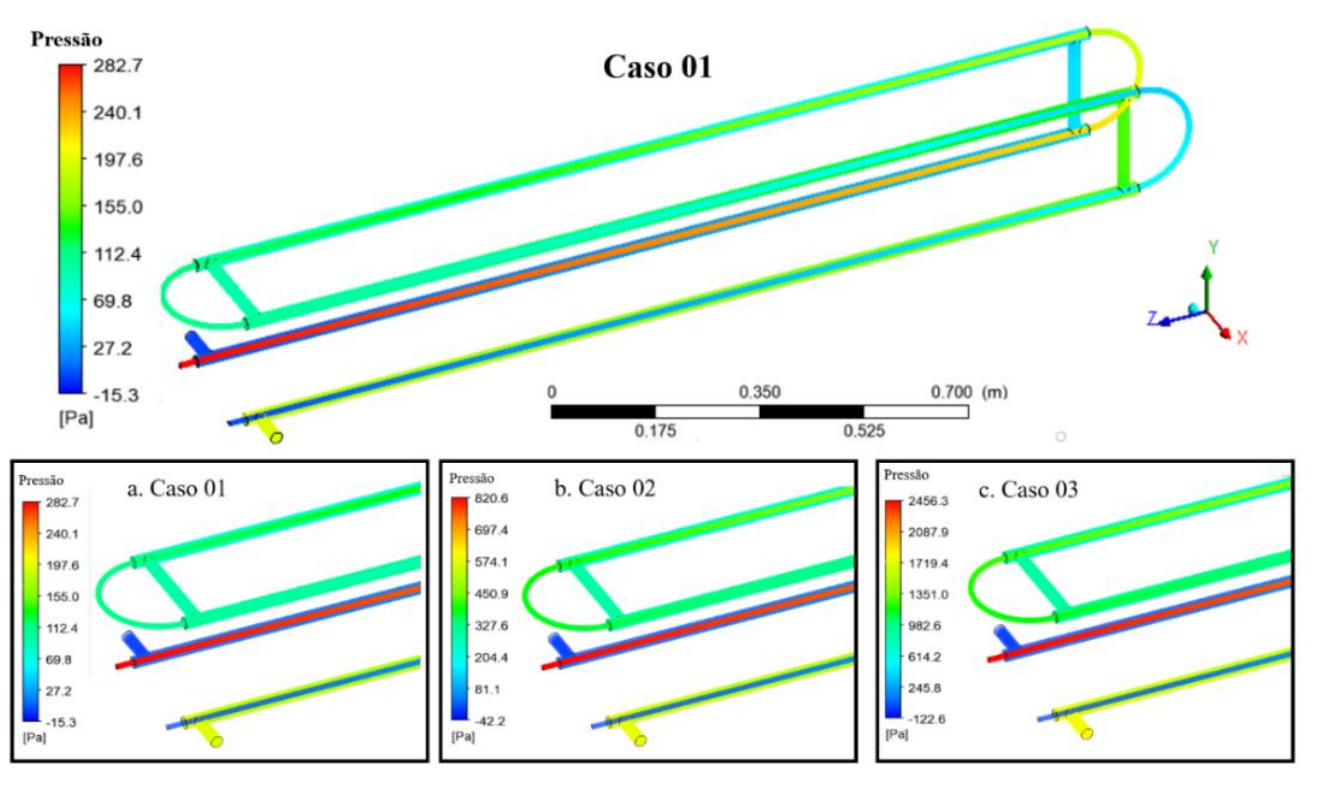

Figura 4 - Perdas de carga da região anular e do tubo para os 4 casos.

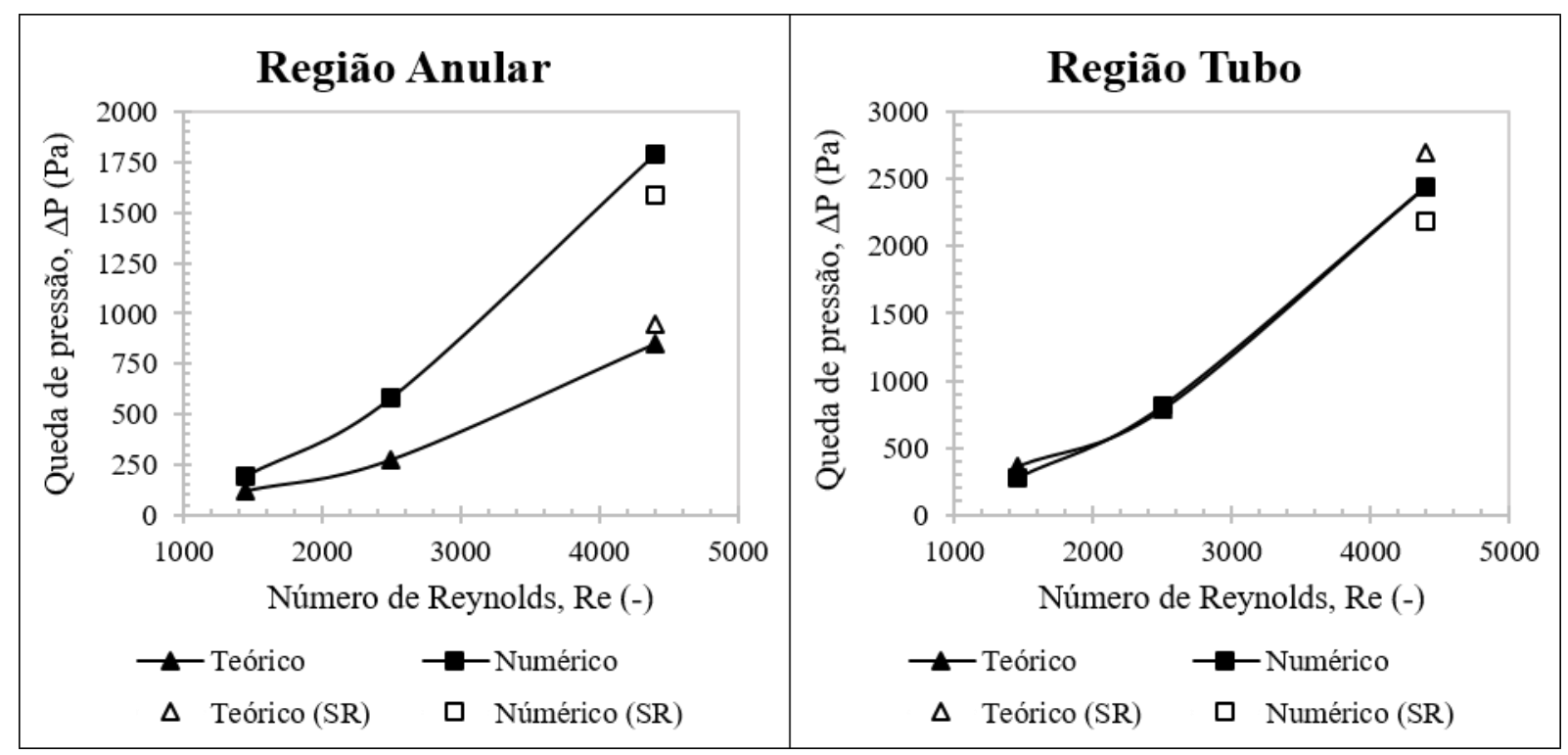




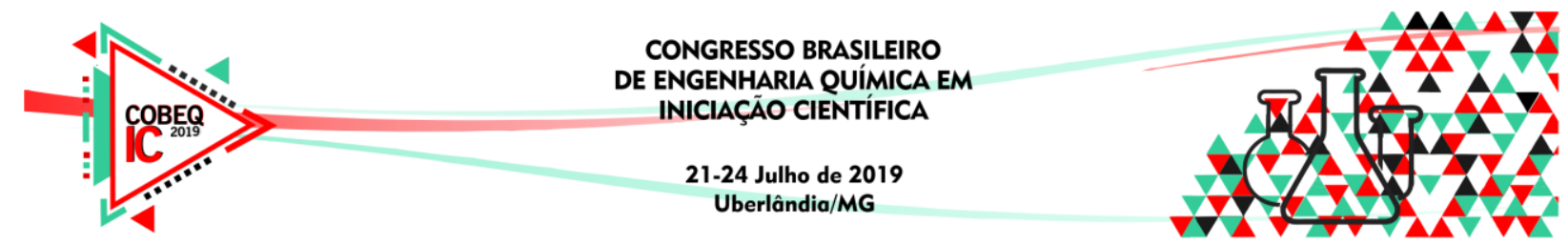

Na Figura 4, as quedas de pressão para diferentes números de Reynolds são comparadas com cálculos teóricos (literatura) e seus desvios padrões são determinados, sendo o maior valor obtido para caso 3 do tubo anular (110\%). Os altos desvios padrões do caso 3 para o tubo anular pode ser inferido devido a região de transição cujo fator de atrito teórico possui um grande erro quando comparado com casos experimentais reais. Enquanto que para o tubo anular no caso 3, pode ser visualizado um desvio padrão também significativo que pode estar associado com uma perda de carga referente a contínuos ciclos de expansões e contrações ao atravessar os acessórios, o qual diminui ao se comparar com um quarto caso onde é desconsiderado a rugosidade do material (SR).

\section{CONSIDERAÇÕES FINAIS}

Os resultados numéricos para a perda de carga obtidos das simulações se demonstram um ótimo modelo para representar o real, apesar da diferença entre a queda de pressão teórica e a numérica essa é ainda bem pequena para uma escala pascal. Concluindo-se que a perda de carga é elevada para situações onde o fluido possui uma baixa temperatura e para regimes de escoamento turbulentos com altas vazões, onde as perdas por atrito e pela mudança repentina da direção são maiores. Conclui-se também que a variação entre a queda de pressão teórica e numérica para o tubo anular decorre da alteração da velocidade do escoamento nos acessórios, em razão da contração e expansão do fluido, acarretando em uma perda de carga maior que a calculada no teórico com velocidade constante.

\section{REFERÊNCIAS}

GOODARZI, M., KHERBEET, A. S., AFRAND, M., SADEGHINEZHAD, E., MEHRALI, M., ZAHEDI, P., DAHARI, M. Investigation of heat transfer performance and friction factor of a counter-flow double-pipe heat exchanger using nitrogen-doped, graphenebased nanofluids. International Communications in Heat and Mass Transfer, 76, 16-23, 2016.

OMIDI, M., FARHADI, M., \& JAFARI, M. A comprehensive review on double pipe heat exchangers. Applied Thermal Engineering, 110, 1075-1090, 2017.

SWAMEE, P. K., AGGARWAL, N., \& AGGARWAL, V. Optimum design of double pipe heat exchanger. International Journal of Heat and Mass Transfer, 51, 2260-2266, 2008.

WANG, W., ZHANG, Y., LEE, K. S., \& LI, B. Optimal design of a double pipe heat exchanger based on the outward helically corrugated tube. International Journal of Heat and Mass Transfer, 135, 706-716, 2019.

YADAV, S., \& SAHU, S. K. Heat transfer augmentation in double pipe water to air counter flow heat exchanger with helical surface disc turbulators. Chemical Engineering and Processing - Process Intensification, 135, 120-132. 2019.

WELTY, J. R, RORRER G. L., FOSTER D. G. Fundamentos de transferência de momento, de calor e de massa. Tradução e revisão técnica Calado V., 6ed. - Rio de janeiro: editora LTC, 2017. 